Objectives: Inflammation is the most important mechanism of plaque disruption playing an essential role in acute coronary syndromes. It is controversial whether the inflammatory mediators are the cause or the result in the development of plaque rupture. Stimulation of interleukins increases adhesion molecules, fibrinogen and plasminogen activator inhibitors, which cause the activation of inflammation and thrombosis. However, the importance of interleukins in acute coronary syndromes has not been clearly defined. We did not find any article concerning relations between the levels of serum interleukin

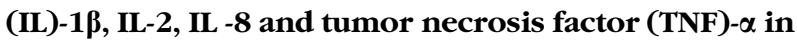
patients with unstable angina pectoris (UAP). So the aim of this study was to determine the levels of serum IL-1 $\beta$, IL-2, IL-8 and TNF- $\alpha$ during the early stage of UAP.

Metbods and results: Thirty-seven patients with UAP (12 females and 25 males; mean age, $57.5 \pm 9.7$ years) within $6 \mathrm{~h}$ of admission and 20 healthy volunteers (eight females and 12 males; mean age, $51.3 \pm 6.3$ years) were included in the study. IL-1ß, IL-2, IL-8 and TNF- $\alpha$ levels were measured using the enzyme-linked immunosorbent assay method. Patients with acute or chronic inflammation, renal failure or chronic heart failure were excluded from the study. The age, gender and risk factors of the study and control groups were

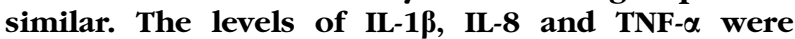
significantly increased $(p<0.0001, p<0.001$ and $p<0.016$, respectively) in patients with UAP. There was no difference of IL-2 levels between the UAP group and controls.

Conclusion: We detected high levels of IL-1ß, IL-8 and TNF- $\alpha$ in patients with UAP during early phase. We suggest that proinflammatory cytokines (e.g. IL-1及, IL-8, TNF- $\alpha$ ) may play an important role in the development of atherosclerosis and its complications.

Key words: Unstable angina pectoris, Inflammation, Interleukins

\section{Levels of serum IL-1及, IL-2, IL-8 and tumor necrosis factor- $\alpha$ in patients with unstable angina pectoris}

Ali Ozeren ${ }^{1, C A}$, Mustafa Aydin ${ }^{1}$, Mehmet Tokac ${ }^{2}$, Nejat Demircan ${ }^{3}$, Murat Unalacak ${ }^{3}$, Ahmet Gurel ${ }^{4}$ and Mehmet Yazici $^{2}$

${ }^{1}$ Department of Cardiology, ${ }^{3}$ Department of Family Medicine and ${ }^{4}$ Department of Biochemistry, Karaelmas University, Faculty of Medicine, Tip Fakültesi, Kardiyoloji Anabilimdali, 67600 Kozlu, Zonguldak, Turkey; ${ }^{2}$ Department of Cardiology, Faculty of Medicine, Selcuk University, Konya, Turkey

\author{
${ }^{\mathrm{CA}}$ Corresponding author \\ Tel: +90 3722610169 \\ Fax: +90 3722610155 \\ E-mail: ozerenali@hotmail.com
}

\section{Introduction}

Atherosclerosis is currently one of the main causes of death. A great majority of deaths related to atherosclerosis occur due to acute complications, mainly based on plaque disruption. ${ }^{1,2}$ Inflammation is the most important mechanism of plaque disruption of which it is crucial to reveal the molecular mechanisms. ${ }^{3,4}$

Previously, the appearance of immune cells at the ischemic site of myocardial tissue was believed to be a response to tissue injury. Inflammatory processes are now recognized to play a central role in the pathogenesis of atherosclerosis and its complications. A growing body of evidence suggests that unstable angina is associated with local and systemic activa- tion of the immune system. Plasma levels of several inflammation markers have been found to be associated with future cardiovascular risk in a variety of clinical settings. These markers include cell adhesion molecules, cytokines, pro-atherogenic enzymes and C-reactive protein (CRP). ${ }^{5}$

Circulating markers may consist of cytokines directly released from inflammatory cells present in the plaques and tissues exposed to recurrent ischemia as well as other reactants produced in response to these cytokines such as adhesion molecules and acute phase proteins. Recent studies suggest that markers of inflammation may reflect different aspects of the atherothrombotic process at different points in the continuum of acute coronary syndromes (ACS). Inflammatory markers have a potential role for the 
prediction of risk for developing coronary artery disease (CAD), and could correlate with severity and future risk for CAD. ${ }^{6}$

Inflammation markers such as CRP, fibrinogen and cytokines have been implicated in the development and progression of $\mathrm{CAD} .^{7-13}$ However, their role in the development of ACS has not been clearly defined. We did not find any article concerning the relations between the levels of serum interleukin (IL)-1 $\beta$, IL-2, IL-8 and tumor necrosis factor (TNF) $\alpha$ in patients with unstable angina pectoris (UAP). So the aim of this study is to determine the levels of serum IL-1 $\beta$, IL-2, IL- 8 and TNF- $\alpha$ during the early stage of UAP.

\section{Materials and methods}

Patients with new onset, Braunwald Class III resting angina within $6 \mathrm{~h}$, but not preceding acute myocardial infarction, were considered as having UAP and were included in the study (12 females and 25 males; mean age, $57.5 \pm 9.7$ years). The study group did not take any medicine and had a fairly unremarkable medical history. Twenty patients (eight females and 12 males; mean age, $51.3 \pm 6.3$ years) with normal coronary arteries on angiogram were considered the control group. Patients with evidence of any infectious disease, significantly higher erythrocyte sedimentation rate $(>20 \mathrm{~mm} / \mathrm{h})$, high fever $\left(\geq 38.3^{\circ} \mathrm{C}\right)$, immunological disorders, neoplastic diseases, chest pain lasting more than $6 \mathrm{~h}$ and normal coronary arteries were not included in the study. Finally, 37 patients remained in the UAP group.

All of the patients with UAP were hospitalized and their medications were initiated accordingly. All patients received acetyl salicylic acid, $\beta$-blocker, weight-adjusted low molecular weight heparin and lipid lowering therapy. Thirty-seven patients enrolled in the study underwent coronary angiography. Significant CAD was defined as more than 50\% narrowing of the luminal diameter in a major epicardial vessel. Blood samples were obtained from the UAP group on admission (within a maximum $6 \mathrm{~h}$ after the onset of pain) before angiographic investigation, to reveal biochemical parameters and interleukin levels. Blood samples were centrifuged at $5000 \mathrm{rpm}$ for 5 min and the collected serum samples were stored at $-80^{\circ} \mathrm{C}$ for a maximum time period of 1 month. IL- $1 \beta$, IL-2, IL- 8 and TNF- $\alpha$ kits were purchased from R\&D ${ }^{\circledR}$ (Roche Diagnostics, USA) and Biosource (USA), and the results were interpreted according to the instructions given by the manufacturer.

All data are presented in the format of the mean \pm standard deviation. Comparisons between two groups were performed using a parametric one-way analysis of variance test. $p<0.05$ was considered statistically significant.

\section{Results}

Age, gender, and risk factors (e.g. smoking, lipid profiles, hypertension, and diabetes mellitus) of the patients are presented in Table 1. There was no statistically significant difference between study and control groups.

In the group of UAP, the levels of IL-1 $\beta$, IL- 8 and TNF- $\alpha$ were significantly increased $(p<0.0001, p<$ $0.001, p<0.016$ and $p<0.05$, respectively) (Table 2 ). There was no difference of IL-2 levels between the UAP group and the control group.

\section{Discussion}

Recently, the role of inflammation in the pathogenesis of atherosclerosis has been understood better. By means of acute phase reactants, interleukins increase both inflammation and smooth muscle hyperplasia. ${ }^{14,15}$ Interleukins, which are soluble hormonelike protein substances, mediate the functions of message production and interaction between immune system cells and determine the immune response.

Stimulation of interleukins increase adhesion molecules, and fibrinogen and plasminogen activator

Table 1. Characteristics of patients

\begin{tabular}{lccc}
\hline & $\begin{array}{c}\text { UAP group } \\
(n=37)\end{array}$ & $\begin{array}{c}\text { Control group } \\
(n=20)\end{array}$ & $p$ value \\
\hline Age (years) & $58 \pm 10$ & $51 \pm 6$ & NS \\
Sex & 25 male/ & 12 male/ & NS \\
Smokers (\%) & 12 female & 8 female & \\
Hypertension (\%) & $24(65)$ & $12(60)$ & NS \\
Diabetes mellitus (\%) & $16(43)$ & $8(40)$ & NS \\
Heredity (\%) & $9(24)$ & $4(20)$ & NS \\
Total cholestrol & $8(21)$ & $3(15)$ & NS \\
Low-density lipoprotein cholestrol & $201 \pm 40$ & $196 \pm 31$ & NS \\
High-density lipoprotein cholestrol & $146 \pm 27$ & $135 \pm 26$ & NS \\
Triglyceride & $32 \pm 6$ & $37 \pm 6$ & NS \\
\end{tabular}

NS, Not significantly different. 
Table 2. Levels of serum IL-1 $\beta, 1 \mathrm{~L}-2,1 \mathrm{~L}-8$ and TNF- $\alpha$

\begin{tabular}{lccc}
\hline & $\begin{array}{c}\text { UAP group } \\
(n=37)\end{array}$ & $\begin{array}{c}\text { Control group } \\
(n=20)\end{array}$ & $p$ value \\
\hline IL-1 $\beta$ (ng/dl) & $378 \pm 34$ & $11 \pm 14$ & $<0.0001$ \\
IL-2 (ng/dl) & $11 \pm 14$ & $15 \pm 19$ & NS \\
IL-8 (ng/dl) & $418 \pm 506$ & $87 \pm 154$ & $<0.001$ \\
TNF- $\alpha$ (ng/dl) & $100 \pm 201$ & $19 \pm 9$ & 0.016 \\
\hline
\end{tabular}

Values are expressed as mean \pm standard deviation. NS, Not significantly different.

inhibitors cause activation of thrombocytes and thus stimulation of thrombosis. ${ }^{16}$ Ischemia causes release of TNF- $\alpha$ and IL- $1 \beta$ from mononuclear cells. It has been proved that there are elevated levels of interleukins in serum of patients with UAP, which carry proinflammatory and procoagulant properties and the degree of elevation of the interleukin level is related to prognosis. ${ }^{17}$

\section{Interleukin-1 $\beta$}

IL-1 $\beta$ is produced largely by activated macrophages, endothelial cells, and also by vascular smooth muscle cells. ${ }^{18,19}$ IL-1 $\beta$ actively participates in the regulation of vascular cell functions, including the stimulation of leukocyte adhesion to the endothelial cells, permeability of vessels, matrix metalloprotease production, suppression of vascular contractility, regulation of the pathways of coagulation within the cell and the production of procoagulator. ${ }^{16}$ It is clear that its levels increase during ischemic heart disease. IL-1 $\beta$ contributes to the development of atherosclerosis. Simon et al $^{20}$ stated that there was a significant increase in IL-1 $\beta$ values in UAP patients compared with stable angina patients.

We found statistically significant increase in IL-1 $\beta$ levels of the study group. The effect of IL- $1 \beta$ on acute process was not clear. IL-1 $\beta$ might be responsible for plaque rupture and triggering ACS. However, IL-1 $\beta$ could be released from the vascular endothelium or ischemic myocardium. Since we could measure IL-1 $\beta$ only at admission, we could not differentiate the source of this increase. In addition to CRP evaluation, we suggest that IL-1 $\beta$ levels could be used for detection of high-risk patients.

\section{Interleukin-2}

In normal conditions immunologic response does not show IL-2 within circulation. IL-2 causes angiogenesis. All IL-2, IL-4, IL- 6 and TNF- $\alpha$ can induce release of IL-2 and its receptors. IL- 2 has a central role in the development of cell-mediated immunity and also it serves as a key factor in the induction of a complex network of cytokines. ${ }^{21}$

Mazzone et $a l .{ }^{22}$ showed that IL-2 levels increased in patients with CAD, but no significant increase was observed in ACS. Takeshita et al ${ }^{23}$ found that serum IL-2 receptors were significantly lower in ACS compared with control. Mizia-stec et al. reported that patients with $\mathrm{CAD}$, irrespective of the form of the disease, have higher serum levels of pro-inflammatory and anti-inflammatory cytokines than control subjects. Increased concentrations of IL-2 in UAP may suggest additional immunologic activation. ${ }^{24}$ On the contrary, levels of IL-2 and IL-2 receptors were reported to be elevated significantly in patients with stable angina, but not in patients with UAP. ${ }^{25}$

In our study, the UAP group revealed a lower but not statistically significant level of IL-2 compared with the control group. This result seems to be in accordance with the study of Simiti et al. ${ }^{25}$ Since IL2 is an anti-inflammatory cytokine, we thought that it might be downregulated first, and later on reaches near normal levels in the early stages of ACS. We measured IL-2 levels only at admission, so we cannot estimate the profile of IL-2 levels. The role of IL-2 on ACS has not been clearly defined as the results of previous studies are contradictory. Further studies are necessary in this issue.

\section{Interleukin-8}

Mononuclear cells, fibroblasts, endothelial cells, keratinocytes and thrombocytes synthesize IL-8. Its synthesis is stimulated by IL- 1 and TNF- $\alpha$. Chemotactic features of IL- 1 and TNF- $\alpha$ are mediated by IL- 8 , which is a crucial chemokine known to attract and activate neutrophils as well as T lymphocytes, and to control their interaction. There are only a few studies in the literature on the IL- 8 levels in patients with myocardial infarction. Simiti et al. reported that the increased plasma IL-8 levels within the first $24 \mathrm{~h}$ after the spontaneous episode of angina could represent a marker of primary UAP, Braunwald's class III-B. But the plasma IL-8 levels do not increase in stable angina. ${ }^{25}$

Qi et al. demonstrated that plasma IL-8 levels increase as a reflector of the abnormal coagulation activity in patients with UAP after coronary angioplasty. ${ }^{26}$ Miya et al. reported that IL-8 seems to have a role in the pathogenesis of acute coronary artery thrombi. This hypothesis leads us to test those interventions on the influence of IL-8 secretion in the early phase of coronary thrombus formation. ${ }^{27}$ In the literature on the pathologic and biochemical investigations of the atherosclerotic coronary plaques, it has been stated that the level of IL-8 increased as an angiogenic factor. ${ }^{27-29}$

In our study, the levels of IL-8 in UAP cases were found significantly increased compared with normal healthy subjects. Our results are in accordance with literature clues. We suggest that IL-8 has an important role in inflammation and coagulation in UAP cases. We did not conclude any definitive relationship 
between other inflammatory parameters and coagulation status, since we did not measure them.

\section{Tumor necrosis factor- $\alpha$}

TNF- $\alpha$ plays a role in the processes of coagulation, ischemia and reperfusion injury. TNF- $\alpha$ may have a certain role in the development of ischemia by causing release of endothelial adhesion molecules, activation of leukocytes and secretion of thrombocyte activating factors. Endothelial cells are highly sensitive to TNF- $\alpha$. By the effect of TNF- $\alpha$ the production of adhesion molecules and procoagulants increases, whereas the synthesis of protein-C is suppressed. ${ }^{30}$

Proving the high serum levels of TNF- $\alpha$ following acute myocardial infarction observed in animal models, TNF- $\alpha$ has been suspected to be related with acute myocardial infarction pathogenesis. ${ }^{30}$ Koukkunen et al. reported that a 3.5-fold increase was observed in fibrinogen and TNF- $\alpha$. They suggested two underlying sources of events; the first is the 'inflammation' factor that includes CRP, fibrinogen and IL-6, and the second is the 'injury' factor that includes troponin- $\mathrm{T}$, creatine kinase-MB mass and TNF- $\alpha$. Both of these factors were independent predictors of the risk of coronary death and other major coronary events. Both TNF- $\alpha$ and IL- 6 were elevated in the coronary sinus compared with the aortic root in patients with UAP. There is an intracardiac inflammatory response in UAP that appears to be the result of low-grade myocardial necrosis. Mizia-stec et al. stated that serum concentrations of TNF- $\alpha$ were significantly higher in patients with CAD than in the control group. ${ }^{24}$

In our study, TNF- $\alpha$ was significantly higher in the study group than in the control group. Although we did not measure troponin, we speculated that this increase might be associated with microinfarcts in UAP patients. Thus, we thought that elevated TNF- $\alpha$ could be used for risk stratification.

\section{Limitations of this study}

Unfortunately, our study contained a relatively small number of patients to reach an exact conclusion. This situation is valid especially for evaluations of IL-8 and IL-2 levels. Another limitation was that we accepted the chest pain commencement time within $6 \mathrm{~h}$ (approximately $4.8 \pm 1.2 \mathrm{~h}$ ) for the UAP group. Some factors such as taking serum samples at admission of the patients with UAP and changes of cytokines according to time passed, not measuring the troponin level that is an indicator of microinfarcts in patients with UAP and high-risk patients, and not determining its relation to other related parameters are the other restrictions.
In conclusion, we suggest that IL- $1 \beta$, IL- 8 and TNF$\alpha$ are directly involved in the triggering stage of acute coronary events. As for CRP, high levels of inflammatory cytokines such as IL- $1 \beta$ and TNF- $\alpha$ may be used for determining the high-risk patients in UAP.

\section{References}

1. Antman EM, Braunwald E. Acute myocardial infarction. In Braunwald, Zipes, Libby (eds). Heart Disease (6th edition). London: WB Saunders Company, 2001: 1114-1127.

2. Worthley SG, Osende JI, Helft G, Badimon JJ, Fuster V. Coronary artery disease: pathogenesis and acute coronary syndromes. Mt Sinai J Med 2001; 68: $167-181$.

3. Neri Serneri GG, Prisco D, Martini F, et al. Acute T-cell activation is detectable in unstable angina. Circulation 1997; 95: 1806-1812.

4. Liuzzo G, Goronzy JJ, Yang H, Kopecky SL, Holmes DR, Frye RL, Weyand CM. Monoclonal T-cell proliferation and plaque instability in acute coronary syndromes. Circulation 2000; 101: 2883-2888.

5. Caligiuri G, Paulsson G, Nicoletti A, Maseri A, Honsson GK. Evidence for antigen-driven T-cell response in unstable angina. Circulation 2000; 102: $1114-1119$

6. Saadeddin SM, Habbab MA, Ferns GA. Markers of inflammation and coronary artery disease. Med Sci Monit 2002; 8: RA5-RA12.

7. Price DT, Loscalzo J. Cellular adhesion molecules and atherogenesis. Am J Med 1999; 107: 85-97.

8. Hillis GS, Flapan AD. Cell adhesion molecules in cardiovascular disease: a clinical perspective. Heart 1998; 79: 429-431.

9. Koenig W. Haemostatic risk factors for cardiovascular diseases. Eur Heart J 1998; 19: C39-C43.

10. Van Willigan G, Gotter G, Akkerman JW. LDLs increase the exposure of fibrinogen binding on platelets and secretion of dense granules. Arterioscler Thromb 1994; 14: 41-46.

11. Danielsen R, Onundarson PT, Thors H, Vidarsson B, Morrissey JH. Activated and total coagulation factor VII, and fibrinogen in coronary artery disease. Scand Cardiovasc J 1998; 32: 87-95.

12. Ernst E, Koenig W. Fibrinogen and cardiovascular risk. Vasc Med 1997; 2: $115-125$.

13. Ho $\mathrm{CH}$, Wang SP, Jap TS. Hemostatic risk factors of coronary artery disease in the Chinese. Int J Cardiol 1995; 51: 79-84.

14. Weissberg P. Mechanisms modifying atherosclerotic disease - from lipids to vascular biology. Atherosclerosis 1999; 147: S3-S10.

15. Mulvihill N, Foley JB, Ghaisas N, Murphy R, Crean P, Walsh M. Early temporal expression of soluble adhesion molecules in patient with unstable angina and subendocardial myocardial infarction. Am J Cardiol 1999; 83: $1265-1267$.

16. Oemar BS. Is interleukin-1 beta a triggering factor for restenosis? Cardiovasc Res 1999; 44: 17-19.

17. Ross R. Atherosclerosis is an inflammatory disease. Am Heart J 1999; 138: S419-S420.

18. Paraskevas F. Cell interactions in the immune response. In: Lee GR, Foerster J, Lukens J, Paraskevas F, Greer JP, Rodgers GM, eds. Wintrobe's Clinical Hematology, Egypt: Williams\&Wilkins, 1999: 564-566.

19. Galea J, Armstrong J, Galea J, et al. Interleukin-1 beta in coronary arteries of patient with ischemic heart disease. Arterioscler Thromb Vasc Biol 1996; 16: 1000-1006.

20. Simon AD, Yazdani S, Wang W, Schwartz A, Rabbani LE. Circulating levels of IL-1beta, a prothrombotic cytokine, are elevated in unstable angina versus stable angina. J Thromb Thrombolysis 2000; 9: 217-222.

21. Simon AD, Yazdani S, Wang W, Schwartz A, Rabbani LE. Elevated plasma levels of interleukin-2 and soluble IL-2 receptor in ischemic heart disease. Clin Cardiol 2001; 24: 253-256.

22. Mazzone A, De Servi S, Vezzoli M, et al. Plasma levels of interleukin 2,6,10 and phenotypic characterisation of circulating T Lymphocytes in ischemic heart disease. Atherosclerosis 1999; 145: 367-374.

23. Takeshita S, Isshiki T, Ochiai $\mathrm{M}$, et al. Systemic inflammatory responses in acute coronary syndrome: increased activity observed in polymorphonuclear leukocytes but not T lymphocytes. Atherosclerosis 1997; 135: $187-192$.

24. Mizia-Stec K, Mandecki T, Zahorska-Markiewicz B, et al. Selected cytokines and soluble forms of cytokine receptors in coronary artery disease. Eur J Intern Med 2002; 13: 115-122.

25. Simiti A, Vida-Simiti L, Cristea A, Olinic N. Increased plasma levels of interleukin-8 in patients with unstable angina pectoris. Rom J Intern Med 1998; 36: 47-56

26. Qi X, Peng Y, Gu J, Li S, Zheng S, Zhang J, Wang T. Inflammatory cytokine release in patients with unstable angina after coronary angioplasty. Jpn Heart J 2002; 43: 103-115.

27. Miya Y, Kanda T, Tamura J, Sumino H, Kurabayashi M. A new murine model of coronary artery thrombosis and role of interleukin- 8 in the 
development of coronary thrombosis. Res Commun Mol Pathol Pharmacol 2001; 108: 108-115.

28. Zhou RH, Shi Q, Gao HQ, Shen BJ. Changes in serum interleukin- 8 and interleukin-12 levels in patients with ischemic heart disease in a Chinese population. J Atheroscler Thromb 2001; 8: 30-32.

29. Li D, Zhao L, Liu M, Du X, Ding W, Zhang J, Mehta JL. Kinetics of tumor necrosis factor alpha in plasma and the cardioprotective effect of a monoclonal antibody to tumor necrosis factor alpha in acute myocardial infarction. Am Heart J 1999; 137: 1145-1152
30. Koukkunen H, Penttila K, Kemppainen A, Halinen M, Penttila I, Rantanen T, Pyorala K. C-reactive protein, fibrinogen, interleukin-6 and tumour necrosis factor-alpha in the prognostic classification of unstable angina pectoris. Ann Med 2001; 33: 37-47.

Received 5 September 2003

Accepted 22 September 2003 


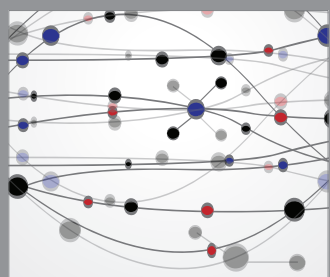

The Scientific World Journal
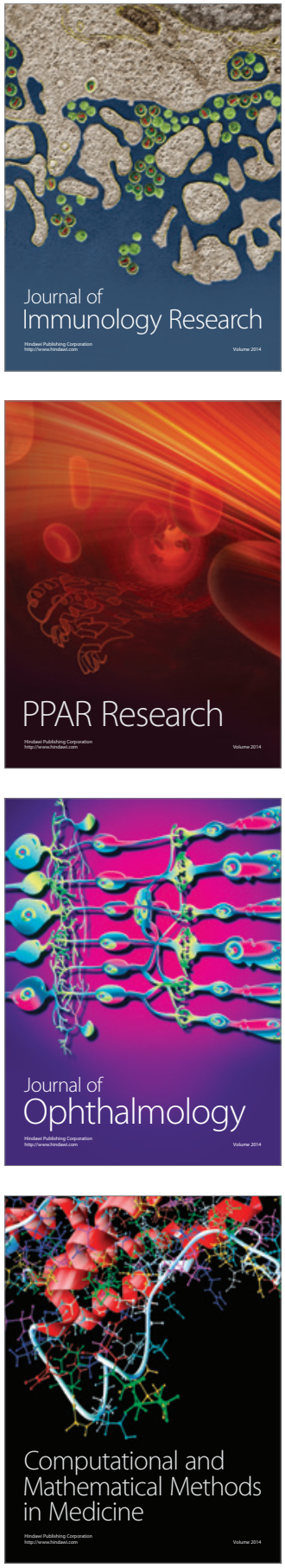

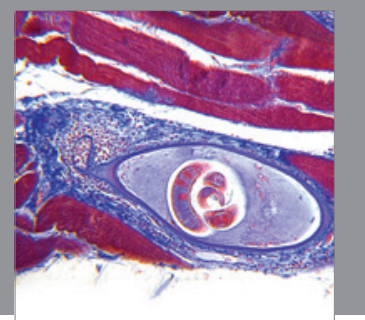

Gastroenterology

Research and Practice
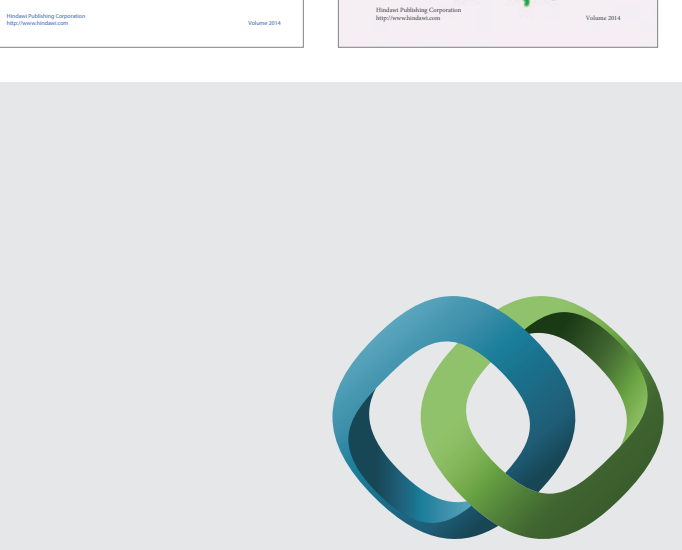

\section{Hindawi}

Submit your manuscripts at

http://www.hindawi.com
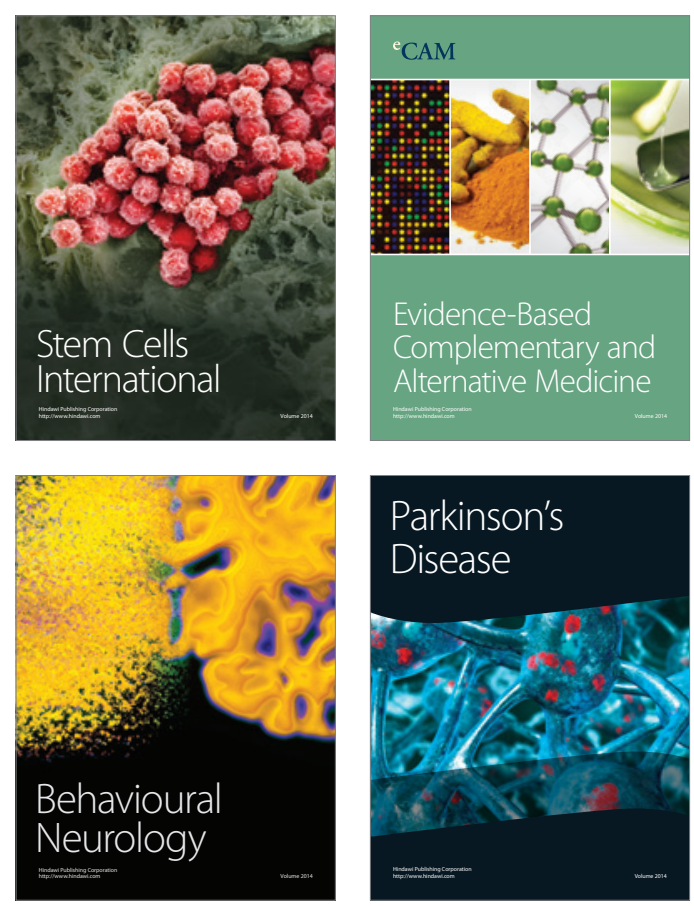

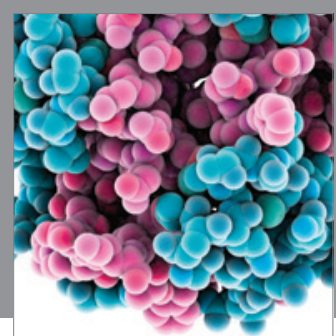

Journal of
Diabetes Research

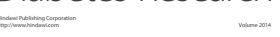

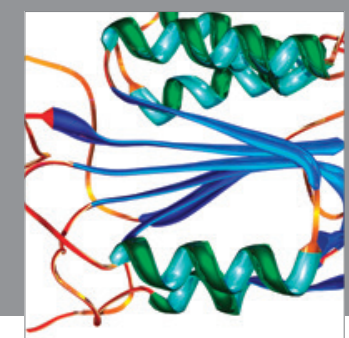

Disease Markers
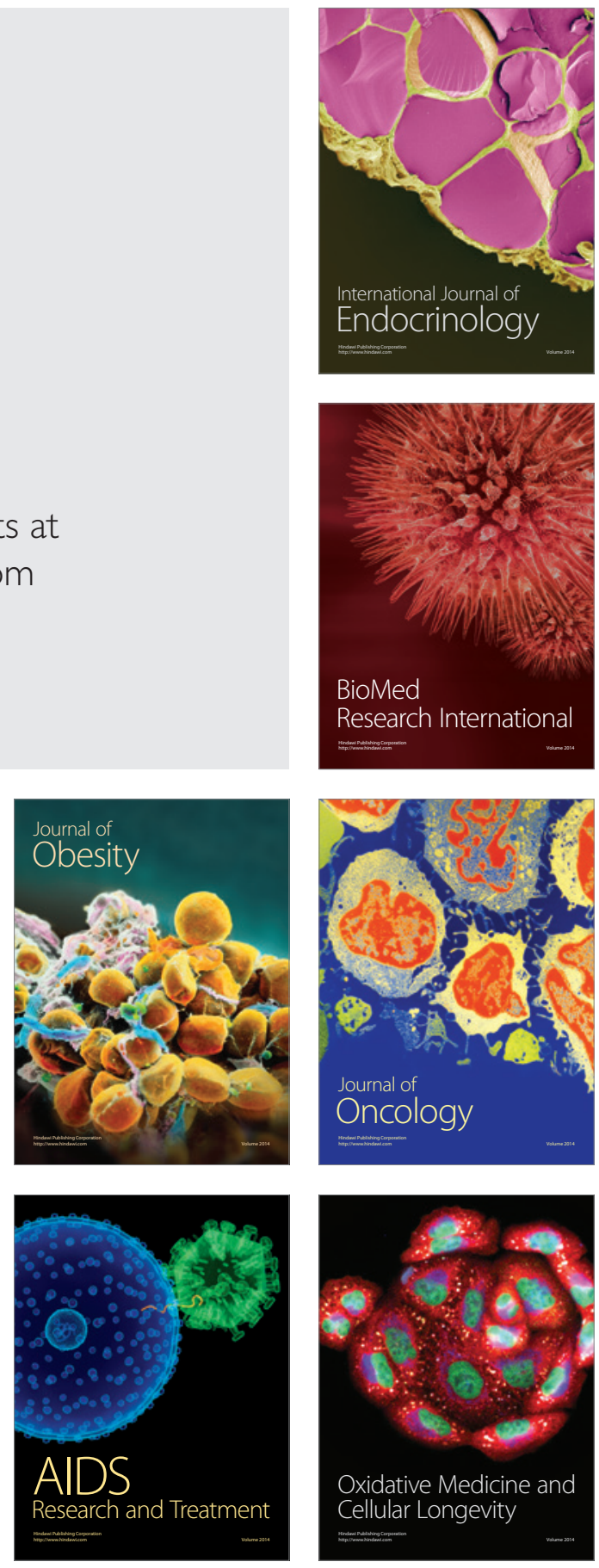